\title{
Calculation and Analysis of Electron Trajectories in a Photocathode Electron Projec- tion Reduction System
}

\author{
L. P. Ku \\ Princeton Plasma Physics Laboratory \\ P. O. Box 451, Princeton, NJ 08543 \\ and \\ P. M. Platzman and G. F. Saville \\ AT\&T Bell Labs \\ Murray Hill, NJ 07974
}

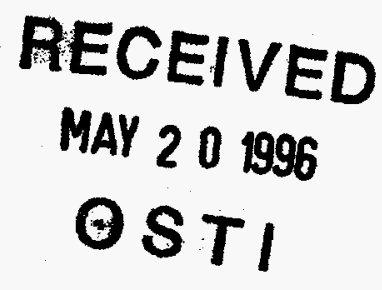

\begin{abstract}
$\underline{\text { Abstract }}$
This report provides a summary of the work performed under the "quick response CRADA" PPPL-95-006. The purpose of the CRADA is to investigate the feasibility of pattern reduction in the photoelectron projection lithography using converging magnetic fields. We analyzed and numerically simulated the trajectories of electrons in a realizable system. We show magnet and electrical grid configurations capable of projecting and reducing images with feature sizes on the order of $0.1 \mu \mathrm{m}$. Distortions less than $10^{-6}$ and spot sizes $<20 \mathrm{~nm}$ appear to be achievable.
\end{abstract}

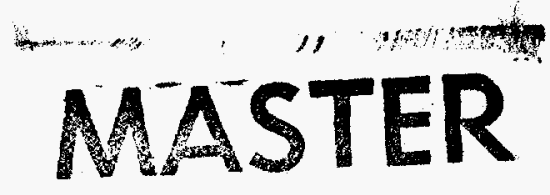




\section{Introduction.}

This report provides a summary of the work performed under a "quick response CRADA" agreement between PPPL and AT\&T Bell Labs (Contract \# PPPL-95-006) entitled "Photocathode Electron Projection Lithography." The purpose of the CRADA was to investigate the feasibility of pattern reduction in the photoelectron projection lithography using a magnetic funnel (MF). In this CRADA agreement, AT\&T was to oversee the development of the program and to provide technical guidance on the systems requirements in the submicron lithography (such as the physical size for coupling with an optical mask); PPPL was to provide technical work in numerical analysis and systems simulation and to attempt a first-cut conceptual design for the magnet system. The overall funding level for this work was $\$ 30 \mathrm{~K}$.

The interest to re-examine projection lithography and to find ways to improve pattern resolution stems from the observation that optical lithography is rapidly approaching the wavelength limit. To march further down the resolution limit, alternatives, such as $\mathrm{X}$-ray, electron and ion-beam techniques, have been intensely pursued. Projection lithography is an alternative studied in the laboratories starting in the 70's and continuing on until mid-80's.

A 1:1 photocathode electron projector (PCEP), in which electrons are used to transfer patterns from a mask to a resist coated silicon wafer, is conceptually very simple. All the features are written simultaneously, with a parallel cathode and anode in a diode configuration. The cathode is uniformly illuminated at back by UV light. Electrons liberated by the photoelectric effect are emitted from the front surface and accelerated towards the anode. A magnetic field parallel to the electric field provides the necessary focusing of the trajectories. Early studies had focused on resolving issues such as proximity effects and image uistortion, primarily at low magnetic focusing field and high accelerating voltage [1,2]. A number of factors were identified that discouraged further applications of this technique, notably the photocathode surface contamination, reliability of uniform photoemission over long exposure time, and the difficulties of making large mask with micron-sized features. In recent years, Saville et al. at AT\&T Bell Labs were able to surmount the surface contamination by using gold-coated mask and the proximity problem by lowering accelerating voltages (to $\sim 3 \mathrm{keV}$ ) [3]. 


\section{DISCLAIMER}

Portions of this document may be illegible in electronic image products. Images are produced from the best available original document. 

With the advent of more sophisticated magnet technology, particularly in the Nuclear Magnetic Resonance (NMR) imaging, magnets of very high fields with remarkable uniformity can now be easily fabricated using superconductors. By using a second set of magnets at the wafer to produce a stronger field, an additional factor of 2 to 3 in image reduction can be achieved in the photoelectron projection method by forcing electrons moving adiabatically along converging magnetic field lines. In this system the photoemitted electrons are accelerated to several $\mathrm{keV}$ by a grid placed at a few cyclotron orbits away from the photocathode in a uniform magnetic field. On passing through the grid the electrons enter an electric field-free region in which the magnetic field strength is increased at the end where the resist coated silicon wafer is placed. In such an electric field free region, the gyrocenter of an electron remains tied to a single field line in the adiabatic limit and the electrons spiral around the converging field lines, resulting in an image demagnification proportional to the square root of the initial to final magnetic field ratio. Such a triode-like system could be used to make large area masks by marrying a conventional optical front end with a PCEP with image demagnification. This system may also be used as a stand-alone lithographic machine.

For this concept to work, it is necessary to estimate the resolution and distortion of the transferred pattern. The objective of this quick response CRADA is to, by direct numerical simulation of electron trajectories, verify the concept and study the resolution and distortion in relation to the magnetic and electric field qualities, thereby providing a basis for possible construction of a prototype system. Factors affecting the quality of pattern transfer due to structural (coil misalignment), mechanical (electrostatic pressure on grid and bending), thermal (mask heating and buckling), and environmental (coil leads, earth magnetic field, etc.) considerations are not discussed in this report. Also, we have not examined issues related to the photocathode and electron resists in this work.

The numerical program constructed selects stochastically the initial velocity and position and then tracks deterministically the subsequent motion of an electron in three-dimensional magnetic and electric fields. We have investigated electron trajectories in a wide range of initial velocity distributions, accelerating voltages and initial and final magnetic field intensities in an axi-symmetric geometry. Scalings using the first-order, single particle orbit theory have been developed to guide the study. Magnetic and electric grid configurations have been found which are capable of projecting and reduc- 
ing several $\mathrm{cm}^{2}$ images with features in the $0.1 \mu \mathrm{m}$ range. Distortions are generally less than 1 part in a million. There is a good depth of focus $(\geq 1 \mu)$, and spot sizes less than $20 \mathrm{~nm}$ are achievable.

In the following, we present a summary of physical analysis in section II and a numerical example for a 2:1 system in section III. More detailed discussions of photoemission process and electron motions in non-uniform electric and magnetic fields as related to a PCEP system maybe found in Ref. 3,4,5 and 6. Figure 1 shows a schematic of an axi-symmetric PCEP pattern reduction apparatus.

\section{Physical Analysis}

The quality of pattern transfer (mapping of a point from photocathode to wafer) in a PCEP system with converging magnetic fields is affected by: (1) the electron initial thermal velocity, (2) the non-adiabaticity due to the magnetic field gradient, (3) the non-uniformity of the magnetic fields at the cathode and wafer, and (4) the transverse electrical field. We provide a brief discussion for each of the factors below.

\section{A. Electron Random Velocity.}

Electrons emitted with different energies perpendicular to the cathode surface will have different focal distance. The spread in focal distance, together with the random initital transverse velocity, causes a point on the photocathode to be mapped as an area on the wafer. The electron velocity perpendicular to the photocathode in a slowly varying magnetic field can be written as

$$
\begin{aligned}
v_{\perp}(z) & =v_{a}+\Delta v_{\perp} \\
& \approx\left(\frac{2 e V}{m}\right)^{1 / 2}\left\{1+\frac{1}{2} \frac{E_{0}}{e V}\left[1-\frac{E_{\|}}{E_{0}}\left(\frac{B(z)}{B_{0}}\right)\right]\right\}
\end{aligned}
$$

where $\mathrm{v}_{\mathrm{a}}$ is the velocity corresponding to the zero emission energy, and $\Delta v_{\perp}$ is the variation due to finite emission energies. $E_{0}$ is the electron initial energy $\left(E_{0}<<e V\right), E_{\|}$is the energy component parallel to the photocathode, $B_{0}$ is the magnetic induction at the photocathode and $B(z)$ is the induction at $z$ (see fig. 1). $\Delta v_{\perp}$ includes two terms corresponding to the effect of non-zero total emission energy and the effect of the transverse component of the velocity. The size of the area on the wafer after $n$ cyclotron 
periods maybe measured by the 'radius of spread', $\Delta r$, as

$$
\Delta r=r_{c}^{0}\left[\frac{\bar{E}}{\mathrm{eV}}\right]\left[\frac{B_{0}}{B_{M}}\right]^{1 / 2} \cdot n .
$$

Alternatively,

$$
\Delta r=\frac{d}{2} \sqrt{\frac{B_{M}}{B_{0}}} \cdot \frac{\bar{E}}{E_{0}} \cdot\left(\frac{E_{0}}{e V}\right)^{3 / 2}\left(\frac{E_{\| l}}{E_{0}}\right)^{1 / 2}
$$

Here, we define $d=z_{w}$-zg, the grid-wafer separation, $B_{M}$ the magnetic induction at the wafer, $V$ the grid voltage, $r_{c}^{0}$ the initial cyclotron radius, and

$$
\frac{\bar{E}}{E_{0}}=1-\frac{E_{\|}}{E_{0}} \cdot \frac{\bar{B}}{B_{0}},
$$

with $\bar{B}$ defined as the average $B$ between the grid and wafer, i. e. $\bar{B} \equiv \int B \cdot d z / d$.

Typically, to ensure sufficient adiabaticity we have $n \sim 10^{4}$ and $\bar{E} / \mathrm{eV} \leq 5 \cdot 10^{-5}$, so that $\Delta \mathrm{r} \leq 20 \mathrm{~nm}$ is achievable for a MF with several Tesla fields. It will produce a transfer and reduction of a pattern with feature size $<0.1 \mu \mathrm{m}$. It is interesting to note that for a fixed demagnification, $\Delta \mathrm{r}$ is inversely proportional to the $3 / 2$ power of the accelerating voltage, but independent of the magnitude of the magnetic induction at the cathode and wafer. Better focusing can be achieved by having 'cold' photoelectrons and higher accelerating voltage. If the initial energy spread is narrow (i. e. $E_{0} \sim$ constant), then for a given demagnification $\Delta \mathrm{r}$ is proportional to the product of $\overline{\mathrm{B}} / \mathrm{B}$ and the grid-wafer separation. Since a larger separation leads to a smaller $\overline{\mathrm{B}} / \mathrm{B}$ when the demagnification is fixed, one can choose a sufficiently large $d$ to ensure adequate adiabaticity without degrading the quality of focusing.

B. Magnetic Field Gradient and Non-Adiabaticity.

To the lowest order of non-adiabatic effects, it is well known that electrons moving in a curved 
field would experience an azimuth drift [7]. The drift causes a rotation which, in a cylindrically symmetric system, is of the form

$$
\Delta \theta \cong \Delta \theta_{0} \cdot\left[1+\gamma\left(\frac{r}{s}\right)^{2}+\cdots\right\}
$$

where $\gamma$ is a constant of order one and $s$ is some distance over which the field changes. The dominant term, $\Delta \theta_{0}$, is a rigid rotation which can be corrected systematically. This rotation is inversely proportional to the magnitude of induction. As pointed out before, the dispersion of a point image, for a given demagnification and grid-wafer separation, does not depend on the magnitude of the induction. The choice of the magnetic induction is largely dictated by reducing this rotation and also by economic considerations. The proper design of the magnet system should minimize the 'non-rigid' terms.

\section{Non-Uniformity in Magnetic Field.}

For pattern transfer over a large area, additional distortions may come from non-uniform magnetic fields across the cathode and wafer surfaces. Such non-uniformity affect the quality of pattern transfer in two ways, non-uniform demagnification and additional de-phasing. The latter can be understood from the fact that sinceand the phase, $\phi$, is

$$
\phi=\frac{\mathrm{e}}{\mathrm{mva}_{\mathrm{a}}} \cdot \int_{\mathrm{z}_{\mathrm{z}}}^{\mathrm{z}_{\mathrm{w}}} \mathrm{B} \cdot \mathrm{ds},
$$

electrons emitted from two points with different radii would have a phase difference when arriving at the wafer:

$$
\Delta \phi=\frac{e}{m v_{a}} \cdot\left\{\int B_{0} \cdot d r-\int B_{M} \cdot d r\right\} .
$$

We note that modern magnets used in the NMR imaging usually achieve uniformity on the order of 
parts in 107. The important consideration here is the proper control of the uniformity in the presence of residual fields from two essentially uniform subsystems.

\section{Transverse Electrical Field from Grid.}

In addition to the non-adiabatic effects due to the field curvature, the transverse electric field from the grid used to accelerate electrons will also introduce distortions. Distortions resulting from the grid can be minimized by properly positioning the grid and using a small mesh spacing. For a grid made of wires parallel to the y-axis, the displacement $\delta y$ due to the $\mathbf{E x B}$ drift, in the limit of $\frac{\omega \cdot \Delta}{\mathrm{v}_{\mathrm{z}}}<2 \pi$, is of the form

$$
\delta \mathrm{y} \cong \kappa \cdot \frac{\mathrm{B}_{0}}{\sqrt{\mathrm{eV}}} \cdot \frac{\Delta^{3}}{\mathrm{Z}_{\mathrm{m}}}
$$

where $\omega$ is the cyclotron frequency, $v_{z}$ is the electron velocity at the grid, $\kappa$ is some constant, $\Delta$ is the mesh half-spacing and $Z_{m}$ is the cathode-grid separation. To minimize the systematic distortion, we should increase the grid voltage, increase the grid-cathode separation, and most importantly, reduce the mesh spacing. However, increasing the grid-cathode separation would increase the image dispersion. Also, if there is an initial energy distribution, there will be a distortion in $x, \delta x$, associated with the 'misplacement' of the grid since the grid can only be at a fixed location corresponding to one particular initial energy [2]. The grid configuration should be such that

$$
\delta \mathrm{r}=\sqrt{\delta \mathrm{x}^{2}+\delta \mathrm{y}^{2}}<\Delta \mathrm{r}
$$

\section{Numerical Analysis}

A. Trajectory Simulation.

Electron trajectories, when interaction effects are ignored, follow the relativistic equation of 
motion (in vector form),

$$
\frac{d v}{d z}=\frac{1}{\sqrt{1-|v|^{2} / c^{2}}} \cdot \frac{e}{m_{0}}\left\{E+v \times B-\frac{E \cdot v}{c^{2}} v\right\} \cdot \frac{1}{v_{z}}
$$

where $m_{0}$ is the electron rest mass and $c$ the speed of light and $v_{z}$ is the $z$-component of the velocity.

Given an initial position and velocity, the positions and velocities at subsequent times may be found by numerically integrating Eq. 10. Here, we chose the initial energy distribution as $p\left(E_{\perp}, E_{\|}\right)=p\left(E_{\perp}+E_{\|}\right) \cdot p(\mu)$, and $p(\mu)=(k+1) \mu^{k}$, where $\mu$ is the cosine angle of the electron velocity vector with respect to the normal of the photocathode. In our study, we used $p\left(E_{\perp}+E_{\|}\right)=\delta\left(E_{\perp}+E_{\|}-E_{0}\right)$ with $E_{0}=0.2 \mathrm{eV}$ and $k=5$ as the reference. This distribution gives $\overline{\mathrm{E}_{11 /} / \mathrm{E}_{0}}=0.25$.

We sampled initial velocity vectors using a random number generator. The trajectories of electrons were followed by integrating Eq. 10 with a 4th order Runge-Kutta integrator. Particular care was taken to minimize computational errors. Typical relative position errors in a constant magnetic field in our calculation were $\sim 10^{-10}$. The magnetic fields at each location were calculated using the MAGLIB routines [8]. These routines are able to rapidly calculate fields in arbitrary 3-dimensional geometries. The positions and velocities at the crossing planes were tallied. Sampling of 30-50 electrons for a given initial position is generally adequate for estimating the average and the standard deviation of the final position.

The standard deviation of the electron radial position exhibits sinusoidal oscillations along the axial locations, following the gyrations of the electrons about the guiding center. The final wafer location (i.e. d) is where the standard deviation is the smallest near the center of the demagnification coils.

\section{B. A 2:1 Pattern Reduction System.}

Here we summarize and discuss a 2:1 reduction system to illustrate the feasibility of designing a magnet system for printing patterns in a circular area of $1.5 \mathrm{~cm}$ radius. This system features $\mathrm{B}_{0}=1 \mathrm{~T}$, 
$B_{M}=4 T, d=0.6 \mathrm{~m}$ and $V=5 \mathrm{keV}$ with the magnetic field essentially constant between the cathode and the grid. Most of the discussions are applicable to similar systems with different design parameters.

\section{Grid}

In [6], we showed that it requires a grid with wire spacing $(2 \Delta)<60 \mu$ in order to achieve resolutions $<20 \mathrm{~nm}$. The grid-cathode separation should be maintained at a distance corresponding to 1 to 2 cyclotron revolutions. For $60 \mu$ spacing, Fig. 2 illustrates the average radial deviation after electrons undergo one additional revolution in $1 \mathrm{~T}$ magnetic field after passing through the grid. Clearly, for grid at 1 or 2 cyclotron revolutions the deviation is mostly due to the initial random energy; grid meshes play an insignificant role in the overall distortion. On the contrary, Fig. 3 shows the deviation when the spacing is doubled. Now the cross-field drift becomes so significant that it is impossible to maintain a good resolution (recall that the deviation is to the 3rd power of $\Delta$ ). In our analysis, we chose $(2 \Delta)=30$ $\mu$ and wire diameters $3 \mu$ arranged parallel to the $y$-axis. The grid was placed near one cyclotron revolution $\left(\mathrm{z}_{\mathrm{g}}=0.7525 \mathrm{~mm}\right)$ where the distortion in $\mathrm{x}$ is minimized [see discussion in ref. 2].

\section{Magnets}

We have chosen a $4 \mathrm{~T}$ demagnification field and an $1 \mathrm{~T}$ background field as the reference. Many techniques exist that can be used to design the magnet system with good uniformity at both the photocathode and the wafer. The following method of decomposition provides a useful guidance relating the image distortion to the field homogeneity requirements. It allows one to make a quick first order design.

In a slowly changing magnetic field the adiabatic invariance of the magnetic moment leads to the conservation of the magnetic flux.

$$
\int_{0}^{r_{1}} B_{0} \cdot d A=\int_{0}^{r_{t}} B_{M} \cdot d A,
$$

where $r_{i}$ and $r_{f}$ are the initial and final radial positions of an electron at the cathode and wafer, respectively. Expanding $B_{0}$ and $B_{M}$ as polynomials of radius variable r, i.e. 
$B_{0}=\sum_{k} B_{0, k} r^{k}$ and $B_{M}=\sum_{k} B_{M, k} r^{k}$, Eq. 11 becomes

$$
\sum_{\mathbf{k}} \frac{B_{0, k}}{k+2} r_{i}^{k+2}=\sum_{k} \frac{B_{M, k}}{k+2} r_{f}^{k+2}
$$

Defining the demagnification $M$ as $M=r f / r_{i}$, we obtain

$$
M \approx \sqrt{\frac{B_{0,0}}{B_{M, 0}}}\left\{1+\frac{1}{B_{0,0}} \sum_{k=2} \frac{B_{0, k}-B_{M, k} M_{0}^{k+2}}{k+2} \cdot r_{i}^{k}\right\}
$$

Eq. 13 can be written as

$$
M=M_{0}\left\{1+\varepsilon_{c}\right\}
$$

where $M_{0}=\sqrt{\frac{B_{0,0}}{B_{M, 0}}}$, is the demagnification when the magnetic fields are uniform at both the cathode and wafer and $\varepsilon \chi$, the second term in the bracket of Eq. 13, is the fractional deviation of $\mathrm{M}$ from M0. For a region of interest where the radii are much smaller than the bore of the magnets, the inhomogeneity of the magnetic fields is typically dominated by the $r^{2}$ and $r^{4}$ terms. In particular, if the demagnification coils are of Helmholtz-type, for which the 2nd order term in the field expansion vanishes, and if the solenoid which provides the background focusing field is very long,

$$
\varepsilon_{c}=\frac{r_{i}^{2}}{4} \cdot \frac{B_{0,2}}{B_{0,0}}+\frac{1}{6} \cdot\left[\frac{B_{0,4}}{B_{0,0}}-M_{0}^{4} \frac{B_{M, 4}}{B_{M, 0}}\right] r_{i}^{4}
$$

Here, the first and second terms are the residue effects at the cathode due to the insertion of the demagnification coil in an otherwise homogeneous solenoid field. For patterning a 1 centimeter-squared mask with distortions $\sim 10 \mathrm{~nm}$, we find $\varepsilon_{\mathrm{c}} \sim 2 \times 10^{-6}$. For coils of Helmholtz-type, this means that the demagnification magnet will have a minimum inner bore of $0.1 \mathrm{~m}$ and possibly a radial build of $0.05 \mathrm{~m}$, 
giving an overall current density of $7 \times 10^{7} \mathrm{~A} / \mathrm{m}^{2}$ for producing the additional $3 \mathrm{~T}$ demagnification field at the center [9].

The residual field from the split coils will make too large a contribution to the overall field nonuniformity at the cathode. A compensation coil may be added at the cathode to cancel such an effect. This added coil, of course, should not generate significant field at the wafer. We found that for a separation between the anode and cathode of $0.6 \mathrm{~m}$ a compensation coil (also a split-type) maybe designed to this effect which carries a current density about 3 orders of magnitude lower than that in the main demagnification coil.

For the solenoid producing $1 \mathrm{~T}$ background field, we chose the one with $0.3 \mathrm{~m}$ inner bore, $3 \mathrm{~m}$ in length and $0.01 \mathrm{~m}$ in radial build with a current density of also $\sim 7 \times 10^{7} \mathrm{~A} / \mathrm{m}^{2}$. The choice of a single long background solenoid simplify the numerical calculation. In practice, one can find designs with a shorter solenoid, but with additional compensation coils that would give equally good field quality by optimization procedures applied to parameter equations such as Eq. 15 [10].

The axial and radial field plots for our sample problem are given in Figs. 4 and 5.

\section{Summary of Results}

Table 1 summarizes the results of simulation using the magnet configuration and optical excitation (with the reference initial conditions) described above. From left to right we give the initial radial position $\mathrm{r}_{\mathrm{i}}$, the final radial position $\mathrm{r}_{\mathrm{f}}$, the average demagnification $\mathrm{M}$, the deviations from the average $\delta \mathrm{M}$, the deviation in angle and finally the magnitudes of magnetic induction. The small effects of the grid were not included in this table. The maximum fractional non-uniformity in the image reduction is $\sim 1.6 \times 10^{-6}$ at the outermost position. The rotation has a constant component of 0.24216 mrad and a small $\mathrm{r}^{2}$ component, the magnitude of which is $\sim 9.15 \times 10^{-3} \mathrm{~m}^{-2}$, or correspondingly, $\gamma=1.6$ for $\mathrm{s}=0.2 \mathrm{~m}$. A typical scatter on the wafer plane for electrons originated at $\mathrm{x}_{\mathrm{i}}=\mathrm{y}_{\mathrm{i}}=1 \mathrm{~cm}$ is shown in Fig. 6. If there were no distortion and defocusing, this would be a single point at $x_{f}=y_{f}=0.502 \mathrm{~m}$. The standard deviation of $\sim 9 \mathrm{~nm}$ due to the initial random velocities is typical for all points along $\mathrm{r}$ and is very consistent with the estimate based on Eq. 3 . 


\section{Conclusion.}

In this CRADA work, we showed that using converging magnetic fields for pattern reduction is a viable option in photocathode electron projection lithography. Numerical simulations of electron trajectories provided us evidence that with properly chosen magnet configurations a near-adiabatic condition could be found. Under such a condition, distortions less than $10^{-6}$ and resolutions $<20 \mathrm{~nm}$ may be achievable in the pattern transfer. The cost of making a 2:1 reduction magnet system, including the cryostat, for further experimental study has been estimated to be $\sim \$ 250-500 \mathrm{~K}$ [11].

In a real system, there will be factors that may limit the attainable resolution with acceptable distortion. There are many challenging technical problems too, such as the grid made of micron-sized wire under very high electrostatic pressures. Nevertheless, we feel that these problems are not insurmountable. With the numerical tool in place, we are now able to investigate the effects of changes in various design parameters and to provide guidance as to how a prototype system should be configured.

\section{Acknowledgments}

This work was supported in part by Stevenson-Wydler (15 USC 3710) Cooperative Research and Development Agreement PPPL No. 95-006 between Princeton Plasma Physics Laboratory under the U.S. Department of Energy Contract No. DE-ACO2-76-CHO3073 and AT\&T Bell Labs, Murray Hill, NJ 07974.

\section{References}

1. Wardly, G. A., J. Vac. Sci. and Tech. 12, No. 6, 1313 (1975).

2. Ward, R., A. R. Franklin, I. H. Lewin, et. al., J. Vac. Sci. and Tech. B 4, No. 1,89 (1986).

3. Saville, G., "Progress Made in Feasibility Stud of 1:1 Photocathode Electron Projection Lithography," un-published AT\&T internal report (1993). 
4. Saville, G., P. M. Platzman, G. Brandes, et. al., J. Vac. Sci. and Tech., To be published.

5. Saville, G., P. M. Platzman, L. P. Ku, J. Vac. Sci. and Tech., B 13, No. 8 (1995).

6. L. P. Ku, "A Note on the Effects of Grid on Electron Trajectories," Princeton Plasma Physics Laboratory Memorandum, un-published (1995).

7. Chen, F. F., Introduction to Plasma Physics, Plenum, NY (1977).

8. Christensen, U. R., "Documentation of EAD Magnetic Codes," Princeton Plasma Physics Laboratory internal report EAD-3705 (1990).

9. Montgomery, D. B., Solenoid Magnet Design, Wiley-Interscience, NY (1969).

10. Williams, J. E. C. (MIT) Private Communication.

11. Dent, P. C. (Everson Electric Company), Wheatney, R (IGC), Williams, J. E. C. (MIT) Private Communications. 


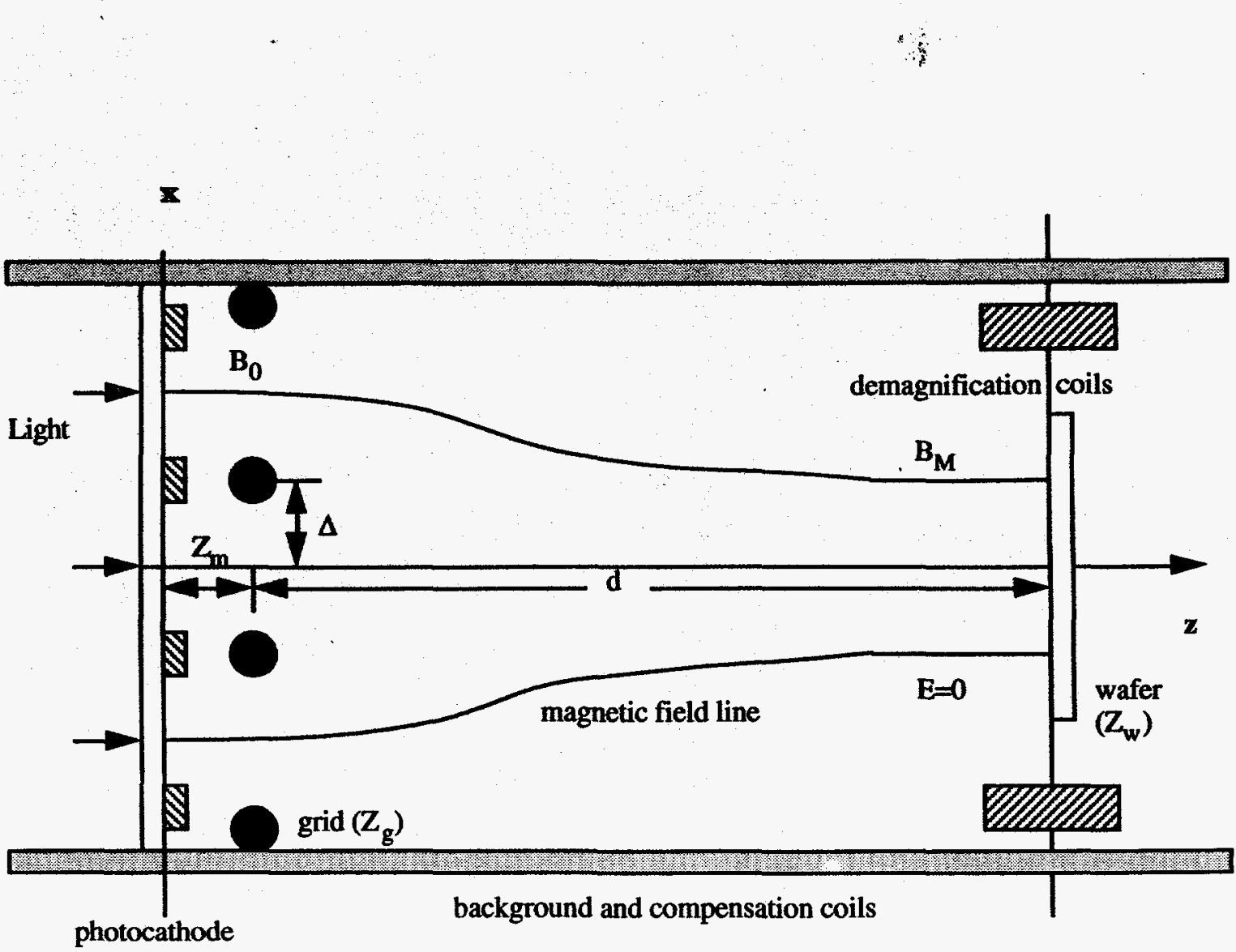

Fig. 1 Schematic showing a photocathode electron projection system for pattern reduction 


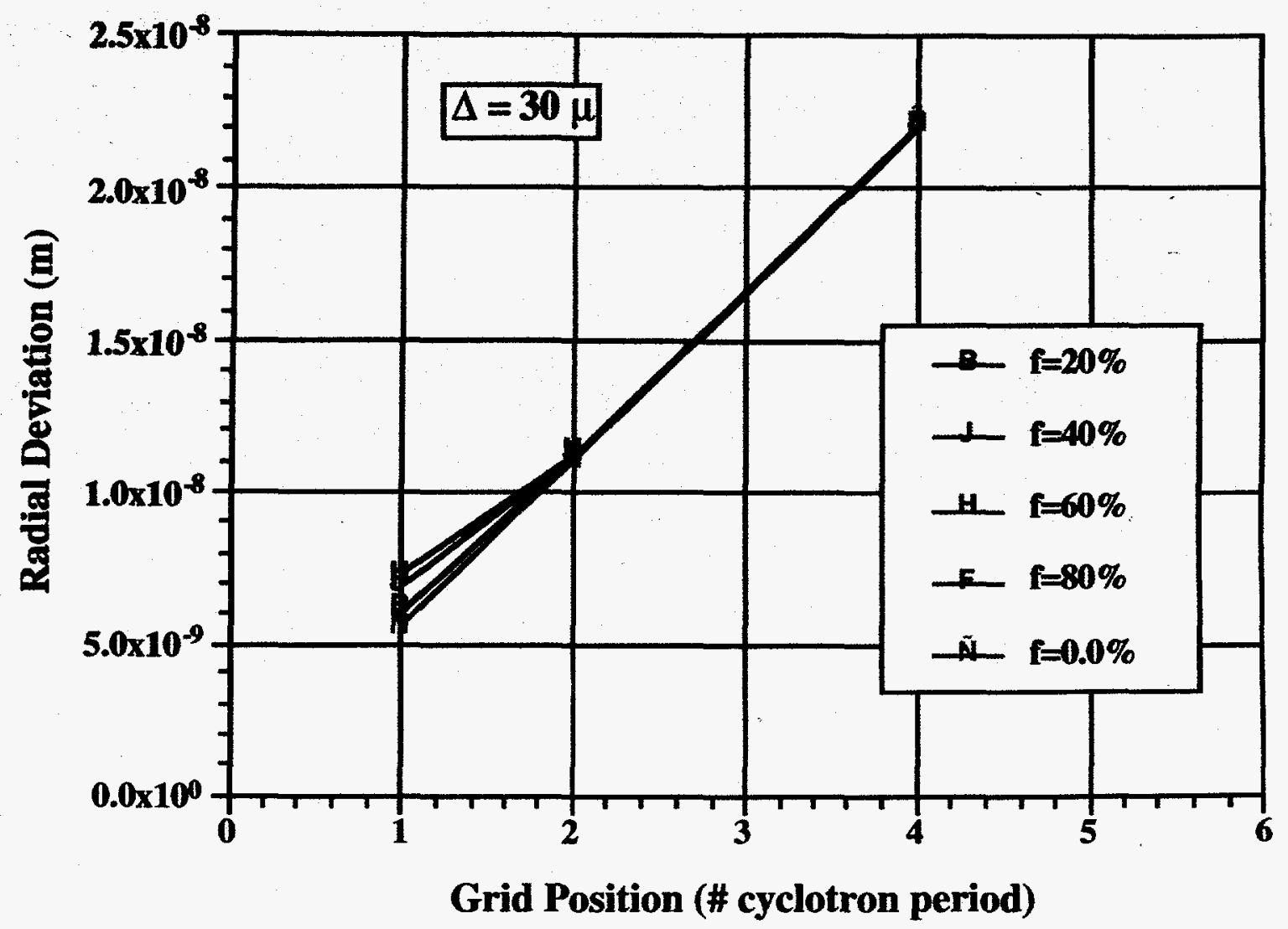

Fig. 2 Average deviation ( $\delta \mathrm{r}$ ) as function of grid position for $\Delta=30 \mu$. Electron initial position: $\mathrm{x}=\mathrm{f} \cdot \Delta$ and $\mathrm{y}=0.0$. 


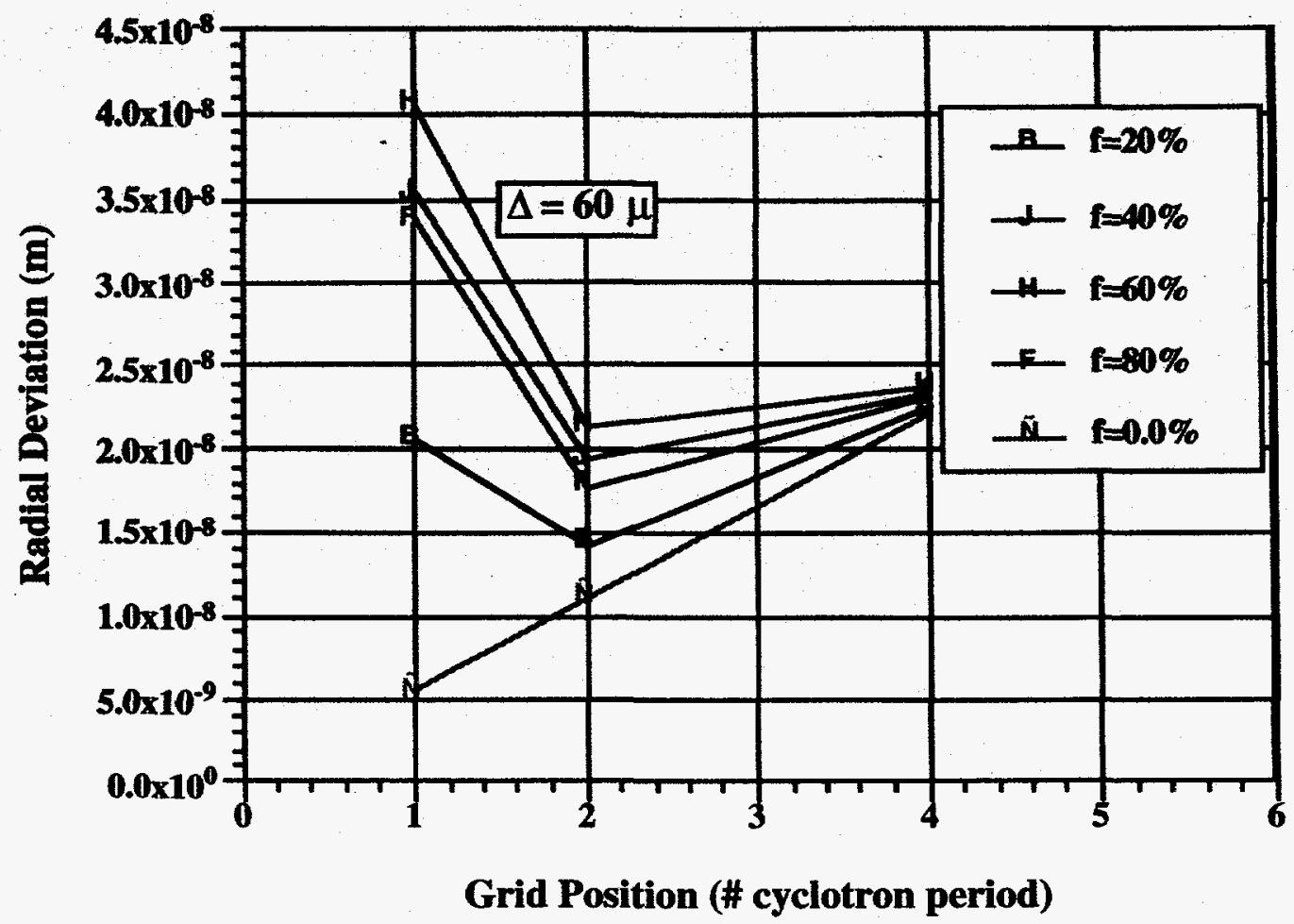

Fig. 3 Average deviation ( $\delta \mathrm{r}$ ) as function of grid position for $\Delta=60 \mu$. Electron initial position: $\mathrm{x}=\mathrm{f} \cdot \Delta$ and $\mathrm{y}=0.0$. 


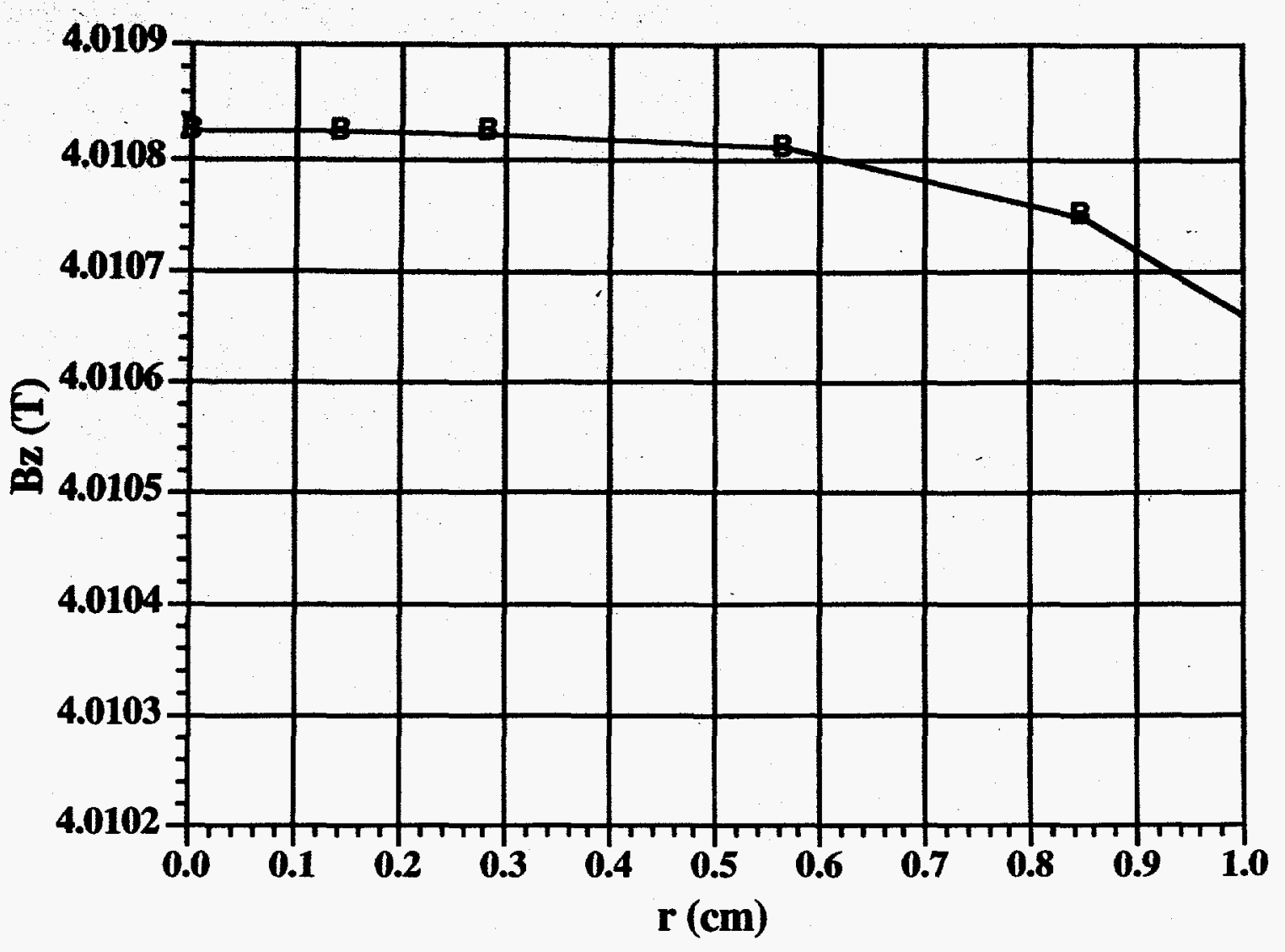

Fig. 4 Plot of $\mathrm{Bz}$ as function of radius at wafer 


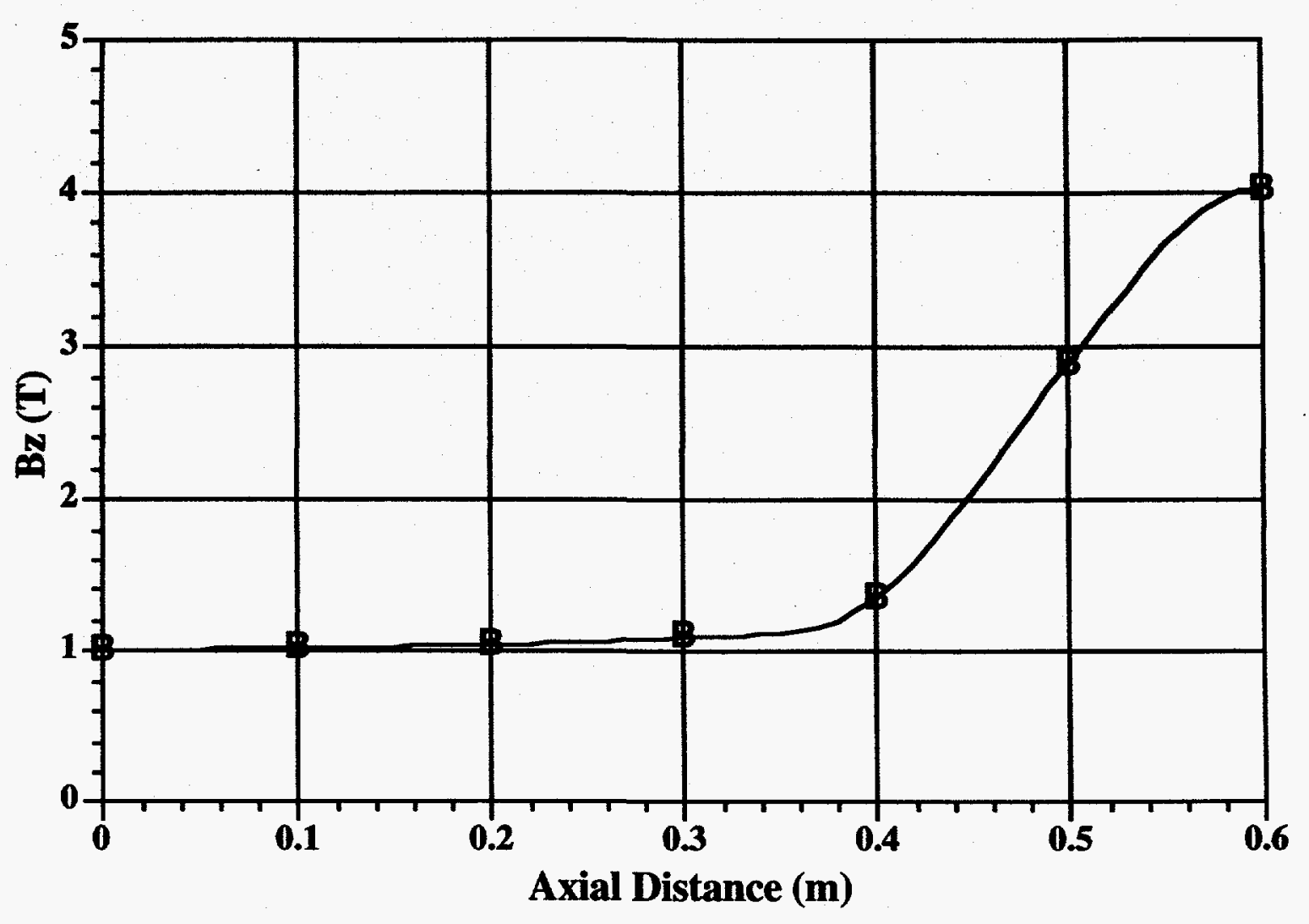

Fig. 5 Plot of the on-axis magnetic field 


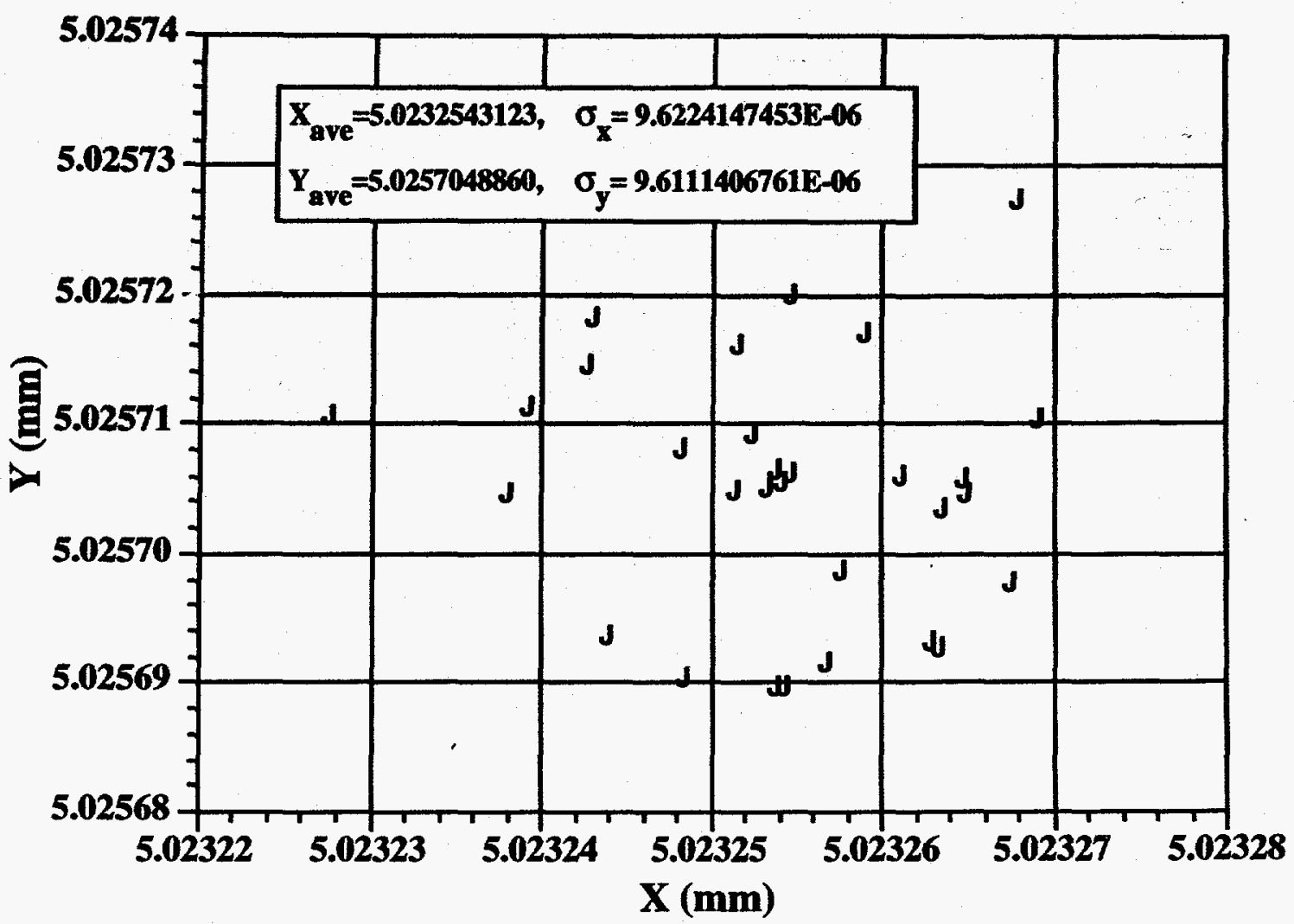

Fig. 6 Pattern of electron spread on the wafer. Electrons originated at $x=1.0 \mathrm{~cm}$, $y=1.0 \mathrm{~cm}$ on the cathode. 


\begin{tabular}{|c|c|c|c|c|c|c|c|c|}
\hline$x_{i}$ & $\mathbf{y i}_{\mathbf{i}}$ & $\mathbf{r}_{\mathbf{i}}$ & $\mathbf{r f}_{\mathbf{f}}$ & $\mathbf{M}$ & $\mathbf{d M}$ & dq & $\mathbf{B}_{\mathbf{z}, 0}$ & $\mathbf{B}_{\mathbf{z}, \mathbf{M}}$ \\
\hline $1.00 \mathrm{E}-04$ & $1.00 \mathrm{E}-04$ & $1.414 \mathrm{E}-04$ & $7.106 \mathrm{E}-05$ & $5.024 \mathrm{E}-01$ & 2.671E-08 & $2.4216 \mathrm{E}-04$ & $1.01255 \mathrm{E}+00$ & $4.01083 \mathrm{E}+00$ \\
\hline $5.00 \mathrm{E}-04$ & $5.00 \mathrm{E}-04$ & $7.071 \mathrm{E}-04$ & 3.553E-04 & $5.024 \mathrm{E}-01$ & $2.653 \mathrm{E}-08$ & 2.4216E-04 & $1.01255 \mathrm{E}+00$ & $4.01083 \mathrm{E}+00$ \\
\hline $1.00 \mathrm{E}-03$ & $1.00 \mathrm{E}-03$ & $1.414 \mathrm{E}-03$ & $7.106 \mathrm{E}-04$ & $5.024 \mathrm{E}-01$ & $2.092 \mathrm{E}-08$ & 2.4218E-04 & $1.01255 E+00$ & $4.01083 \mathrm{E}+00$ \\
\hline $2.00 \mathrm{E}-03$ & $2.00 \mathrm{E}-03$ & $2.828 \mathrm{E}-03$ & $1.421 \mathrm{E}-03$ & $5.024 \mathrm{E}-01$ & $2.488 \mathrm{E}-09$ & $2.4223 \mathrm{E}-04$ & $1.01255 \mathrm{E}+00$ & $4.01083 \mathrm{E}+00$ \\
\hline $4.00 \mathrm{E}-03$ & $4.00 \mathrm{E}-03$ & $5.657 \mathrm{E}-03$ & $2.842 \mathrm{E}-03$ & $5.024 \mathrm{E}-01$ & $5.157 \mathrm{E}-08$ & $2.4244 \mathrm{E}-04$ & $1.01255 \mathrm{E}+00$ & $4.01082 \mathrm{E}+00$ \\
\hline $6.00 \mathrm{E}-03$ & $6.00 \mathrm{E}-03$ & $8.485 \mathrm{E}-03$ & $4.263 \mathrm{E}-03$ & $5.024 \mathrm{E}-01$ & $2.555 \mathrm{E}-08$ & $2.4280 \mathrm{E}-04$ & $1.01255 \mathrm{E}+00$ & $4.01082 \mathrm{E}+00$ \\
\hline 8.00E-03 & $8.00 \mathrm{E}-03$ & $1.131 \mathrm{E}-02$ & $5.685 \mathrm{E}-03$ & $5.024 \mathrm{E}-01$ & 4.661E-07 & $2.4332 \mathrm{E}-04$ & $1.01255 \mathrm{E}+00$ & $4.01081 \mathrm{E}+00$ \\
\hline $1.00 \mathrm{E}-02$ & $1.00 \mathrm{E}-02$ & $1.414 \mathrm{E}-02$ & $7.106 \mathrm{E}-03$ & $5.024 \mathrm{E}-01$ & $1.602 E-06$ & $2.4399 \mathrm{E}-04$ & $1.01255 \mathrm{E}+\infty 0$ & $4.01079 \mathrm{E}+00$ \\
\hline
\end{tabular}

(1) $M=r f / r_{i}, r_{i}, r_{f}=$ initial and final radii (m).

(2) $\mathrm{dM}=11.0-\mathrm{M} / \mathrm{M}_{0} \mathrm{l}, \mathrm{M}_{0}=\operatorname{sqrt}\left(\mathrm{B}_{0}(0) / \mathrm{B}_{\mathrm{M}}(0)\right)$

(3) $\mathrm{dq}=$ angle of rotation in radian

(4) $\mathbf{B}_{\mathbf{Z}, 0}, \mathbf{B}_{\mathbf{Z}, \mathbf{M}}=$ initial and final magnetic field ( $\left.\mathrm{T}\right), \mathrm{z}$ component.

(5) $\mathrm{z}_{\mathrm{w}}=0.599474 \mathrm{~m}$

\section{DISCLAIMER}

This report was prepared as an account of work sponsored by an agency of the United States Government. Neither the United States Government nor any agency thereof, nor any of their employees, makes any warranty, express or implied, or assumes any legal liability or responsibility for the accuracy, completeness, or usefulness of any information, apparatus, product, or process disclosed, or represents that its use would not infringe privately owned rights. Reference herein to any specific commercial product, process, or service by trade name, trademark, manufacturer, or otherwise does not necessarily constitute or imply its endorsement, recommendation, or favoring by the United States Government or any agency thereof. The views and opinions of authors expressed herein do not necessarily state or reflect those of the United States Government or any agency thereof. 